\title{
NONIMMERSIONS OF COMPLEX GRASSMANN MANIFOLDS
}

\author{
by SAMUEL A. ILORI
}

(Received 8th May 1979)

\section{Introduction}

If an oriented manifold $M$ immerses in codimension $k$, then the normal bundle has dimension $k$ such that its Euler class $x \in H^{k}(M ; Z)$ and $\chi^{2} \in H^{2 k}(M ; Z)$. (Cf. (3)).

If $M$ is the complex Grassmann manifold $G_{2}\left(C^{n}\right)$ of 2-planes in $C^{n}(n=$ $4,5, \ldots, 15,17)$, then $\operatorname{dim} M=4 n-8 \equiv d$ and we shall show that although $M$ immerses in $\boldsymbol{R}^{2 d-1}$ by classical results (3), $M$ does not immerse in $\boldsymbol{R}^{d+d / 2}$.

The same result was obtained for $n=4$ and 5 by Connell (2) and for $n=6$ and 7 by the author (6). The nonimmersion results of this paper are new for $n=8,9, \ldots, 15,17$ and they are an improvement over the result for the general $G_{2}\left(C^{n}\right)$ obtained in (5). In this paper, we use generators of the cohomology ring of $G_{2}\left(\boldsymbol{C}^{n}\right)$ different from those used in (2) and (6) and this simplifies the calculations considerably.

\section{2. $G_{2}\left(\boldsymbol{C}^{n}\right)$}

Since the dimension of $G_{2}\left(C^{n}\right)$ is $4 n-8$, it follows that $\chi \in H^{2 n-4}\left(G_{2}\left(C^{n}\right) ; Z\right)$. We shall first investigate the group, $H^{2 n-4}\left(G_{2}\left(C^{n}\right) ; Z\right)$. We denote the Schubert variety, $\Omega_{a_{0} a_{1}}$ by $\left[a_{1}, a_{2}\right]$ and the corresponding Schubert class by $\left[a_{0}, a_{1}\right]^{*}$.

Lemma 2.1. The cohomology group $H^{2 n-4}\left(G_{2}\left(C^{n}\right) ; Z\right)$ is freely generated either by the $(n-1) / 2$ Schubert classes

$$
\left\{[0, n-1]^{*},[1, n-2]^{*}, \ldots,\left[\frac{n-3}{2}, \frac{n+1}{2}\right]^{*}\right\}, \quad \text { if } n \text { is odd }
$$

or by the $n / 2$ Schubert classes

$$
\left\{[0, n-1]^{*},[1, n-2]^{*}, \ldots,\left[\frac{n-2}{2}, \frac{n}{2}\right]^{*}\right\}, \quad \text { if } n \text { is even. }
$$

Moreover, the square of each generator is equal to $[0,1]^{*}$, the generator of $H^{4 n-8}\left(G_{2}\left(C^{n}\right) ; Z\right)$ and any mixed product of two different generators is equal to zero.

Proof. From Theorem II p. 352 of (4), and since

$$
\operatorname{dim}\left[a_{0}, a_{1}\right]=a_{0}+a_{1}-1,
$$


it follows that $H^{2 n-4}\left(G_{2}\left(C^{n}\right) ; Z\right)$ is generated by all Schubert classes

$$
\left\{\left[a_{0}, a_{1}\right]^{*} \mid a_{0}+a_{1}-1=n-2\right\} .
$$

Hence, the first part of the lemma follows. Also from Theorem I p. 327 and Theorem II p. 331 of (4), we have that Schubert varieties of complementary dimension intersect if and only if

$$
a_{1}+b_{0} \geqq n-1 \quad \text { and } \quad a_{0}+b_{1} \geqq n-1 \text {, }
$$

and that the intersection is simple and is at a unique point. Thus the second part of the lemma also follows.

The result of this paper can now be stated as

Proposition 2.2. If $d=4 n-8=\operatorname{dim}\left(G_{2}\left(C^{n}\right)\right)$, and $n=4, \ldots, 15,17$ we have that

$$
G_{2}\left(C^{n}\right) \subseteq R^{2 d-1} \quad \text { and } \quad G_{2}\left(C^{n}\right) \nsubseteq \boldsymbol{R}^{d+1 / 2 d} \text {. }
$$

Proof. The fact that $G_{2}\left(C^{n}\right) \subseteq R^{2 d-1}$ follows from (3). Now the generator $[0,1]^{*}$ of the cohomology group $H^{4 n-8}\left(G_{2}\left(C^{n}\right) ; Z\right)$ is given by

$$
[0,1]^{*}=w_{2, n-2}(1 ; F)=\left[\sigma_{2}\left(\gamma_{0}, \gamma_{1}\right)\right]^{n-2} \text {. }
$$

(Cf. p. 328 of (7), where $\sigma_{2}\left(\gamma_{0}, \gamma_{1}\right)=c_{2}(\gamma)$, the second Chern class of the canonical bundle $\gamma$ over $\left.G_{2}\left(C^{n}\right)\right)$. Hence from Section 3 of (5), it follows that the generator y can be identified as

$$
y=-\sigma_{2}\left(\gamma_{0}, \gamma_{1}\right)
$$

Hence a generator of $H^{4 n-8}\left(G_{2}\left(C^{n}\right) ; Z\right)$ is

$$
[0,1]^{*}=\left[\sigma_{2}\left(\gamma_{0}, \gamma_{1}\right)\right]^{n-2}=(-y)^{n-2} .
$$

Now consider the case when $n$ is odd and assume $M$ immerses in $\mathbf{R}^{d+d / 2}$. Let

$$
\chi=a_{1}[0, n-1]^{*}+a_{2}[1, n-2]^{*}+\ldots+a_{(n-1) / 2}\left[\frac{n-3}{2}, \frac{n+1}{2}\right]^{*}
$$

be the Euler class of the normal bundle. Therefore, $\chi$ is an element of $H^{d / 2}\left(G_{2}\left(C^{n}\right) ; Z\right)$ and by Lemma 2.1 above,

$$
\begin{aligned}
\chi^{2} & =\left(a_{1}^{2}+a_{2}^{2}+\ldots+a_{(n-1) / 2}^{2}\right)[0,1]^{*} \\
& =-\left(a_{1}^{2}+a_{2}^{2}+\ldots+a_{(n-1) / 2}^{2}\right) y^{n-2} .
\end{aligned}
$$

Then by (3), $\chi^{2}=p_{n-2}$ where $p_{r}$ is the $r$-th Pontrjagin class, and from Section 5 of (5) we have

$$
a_{1}^{2}+a_{2}^{2}+\ldots+a_{(n-1) / 2}^{2}=3\left(\begin{array}{c}
3 t-1 \\
t-1
\end{array}\right)\left(\begin{array}{c}
3 t-2 \\
t-2
\end{array}\right) \frac{t^{2}-9 t+6}{(t-1)(3 t-2)},
$$

where $n=2 t+1$. Now the right hand side of the above quadratic equation is negative if and only if $2 \leqq t \leqq 8$, i.e. if and only if $n=5,7,9,11,13,15,17$. In these cases, the quadratic equation has no integral solution. This is a contradiction and so nonimmersion in codimension $2 n-4=d / 2$ is established. 
Similarly, we obtain the following quadratic equation when $n$ is even.

$$
a_{1}^{2}+a_{2}^{2}+\ldots+a_{n / 2}^{2}=\left(\begin{array}{c}
3 t-3 \\
t-2
\end{array}\right)^{2} \frac{t^{2}-8 t+6}{(t-1)^{2}},
$$

where $n=2 t$. The right hand side is negative if and only if $2 \leqq t \leqq 7$, i.e. if and only if $n=4,6,8,10,12,14$. In these cases, the quadratic equation has no integral solution and so nonimmersion in codimension $2 n-4=d / 2$ is also established. This completes the proof of the proposition.

\section{REFERENCES}

(1) A. Borel, Sur la cohomologie des espaces fibrés principaux et des espaces homogènes de groupes de Lie compacts, Ann. of Math. (2) 57 (1953), 115-207.

(2) Fred. J. Connelu, Nonimmersions of low dimensional flag manifolds, Proc. Amer. Math. Soc. 44 (1974), 474-478.

(3) M. Hirsch, Immersions of manifolds, Trans. Amer. Math. Soc. 93 (1959), 242-276.

(4) W. V. D. Hodge and D. Pedoe, Methods of algebraic geometry, Vol. II (Cambridge Univ. Press, New York, 1952).

(5) S. G. Hoggar, A nonembedding result for complex Grassmann manifolds, Proc. Edinburgh Math. Soc. 17 (1970), 149-153.

(6) S. A. ILORI, Nonimmersions of flag manifolds, Proc. Amer. Math. Soc. 61 (1976), 141-144.

(7) A. W. Ingleton, Tangent flag bundles and generalized Jacobian varieties. Accad. Naz. Lincei Rend. Cl. Sci. Fis. Mat. Natur. 8 (1969), 323-329, 505-510.

Department of Mathematics

UNIVERSTTY OF IBADAN

IBADAN, NigERIA 